\title{
Proposal of a Sensing Circuit for Detecting Physical Quantity Regardless of Electrical Units
}

\author{
Kei Sato ${ }^{\text {a,*, Seiichi Serikawa }}{ }^{\text {a }}$ \\ ${ }^{a}$ Department of Electrical and Electronic Engineering, Kyushu Institute of Technology, Kitakyushu, Japan \\ *Corresponding Author: satou.kei991@mail.kyutech.jp
}

\begin{abstract}
In recent years, the introduction of IoT with electronic devices has progressed rapidly, and various kinds of sensors are widely used in electrical appliances and home appliances. It is necessary to prepare a number of sensor-dependent detection circuits which measure the output of each sensor because the quantity of electricity depends on the sensor. This is a bottleneck in achieving price reduction. Since different sensors are used in different ways, repair and replacement in case of failure vary depending on the type of sensors. To solve those problems, a smart sensing circuit for versatilely detecting the different quantity of electricity is proposed not depending on the sensor type. In this paper, the sensor-independent sensing circuit is proposed to uniformly detect the electric quantities of voltage, current, and resistance. The proposed circuit achieves both sensorindependent usage and low cost. It is also possible to be easily and promptly replaced regardless of the sensor type when a malfunction occurs.
\end{abstract}

Keywords: Sensor, Detection, Sensor-independent circuit, Unit

\section{Introduction}

In recent years, the demand for sensors has been increasing as IoT technologies progress [1]. There are various types of sensors such as temperature sensors, optical sensors, and distance sensors. Button switches installed in most electric appliances are also a type of sensor. The sensors are the devices that convert any physical quantities such as heat, light, temperature, and force into electrical quantities such as voltage, current, and resistance. There are different types of sensors that measure the same physical quantity. For example, thermocouples, thermistors, and far-infrared sensors are available for sensing temperature. Optical sensors include photodiodes, $\mathrm{CdS}$ cells, and photo-multiplier. The output of such sensors is the quantity of electricity such as voltage, current, resistance, capacitance, and inductance. The sensor outputs vary in a wide range depending on the type of sensor. In the case of the photodiode, the output current varies greatly in proportion to the intensity of light from $\mathrm{nA}$ to $\mathrm{mA}$. Therefore, a suitable detection circuit should be designed depending on both the type of sensor and the measurement range. It is expensive because it cannot be mass-produced. The sensor-dependent made-to-order detection circuit cannot be replaced immediately when a malfunction occurs, and their usages depend on the type of sensor.

This study aims to develop a sensor-independent circuit that can measure the sensor output with an identical circuit, regardless of the unit of electric quantity provided by sensors. In this study, we propose a unique circuit that can detect electric quantities of voltage, current, and resistance. The proposed circuit can be applied to a variety of sensors. It has an advantage in cost reduction and common use over the sensor-dependent circuits.

\section{Principle}

\subsection{Inverting amplifier circuit}

The circuit proposed in this study employs an inverting amplifier circuit using an operational amplifier. The operational amplifier, which is an integrated circuit that incorporates a differential amplifier circuit, level shift circuit, and so on., has an extremely large voltage amplification factor. An ideal operational amplifier has the characteristics that input impedance is equal to infinity and output impedance is equal to 0 . When it is used for negative feedback, the inverting input terminal and non-inverting 
input terminal equal to imaginary short-circuited, and the voltage between them is almost zero [2].

Figure 2.1 shows an inverting amplifier circuit. As for a phase of output voltage $V_{\text {OUT }}$, it is inverted that of the input voltage $V_{\mathrm{IN}}$. The amplification factor is determined by the values of $R_{1}$ and $R_{2}$. The reference voltage $V_{\mathrm{S}}$ corresponds to analog ground, that is, $0 \mathrm{~V}$.

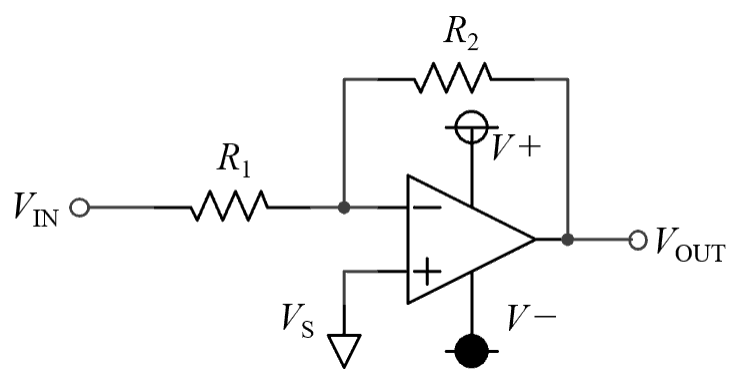

Fig. 2.1 Inverted amplifier circuit.

\subsection{Detection principle}

In this study, it is assumed that the units of electricity obtained by sensors are voltage, current, and resistance. When a sensor is connected to the detection circuit, a user does not need to be aware of the type of sensor output. The output of the common detection circuit changes when the input value of the sensor changes without the prior information on the units of the sensor.

Figure 2.2 shows the voltage detection circuit. Let the input voltage $V_{\mathrm{IN}}$ be the voltage generated by a sensor. In the case of thermocouples, the input voltage $V_{\mathrm{IN}}$ changes depending on the temperature. The switch in the upper left of Fig. 2.2 is connected to $V_{\mathrm{S}}$. As a result of considering the characteristics of an operational amplifier that input impedance is infinite and two input terminals are imaginary short-circuited, the relationship between output voltage $V_{\text {OUT }}$ and the input voltage $V_{\mathrm{IN}}$ is represented in Equation (2.1). In

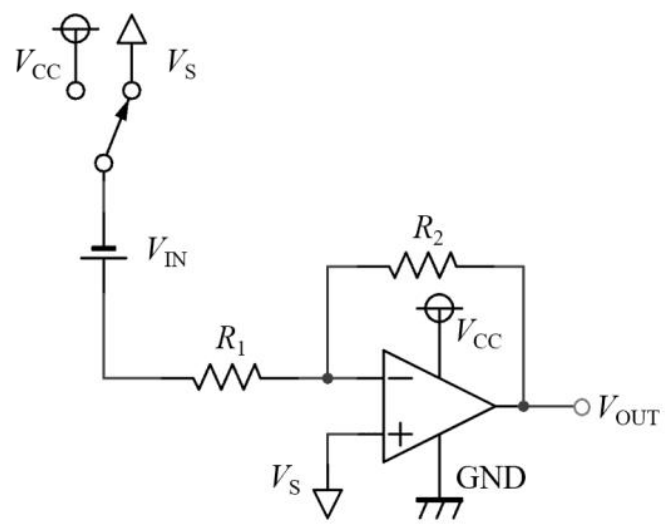

Fig. 2.2 Voltage detection circuit.
Eq. (2.1), the amplification factor is $R_{2} / R_{1}$ and $V_{\text {OUT }}$ changes with the change of $V_{\mathrm{IN}}$.

$$
V_{\text {OUT }}=-\frac{R_{2}}{R_{1}} V_{I N}+V_{S}
$$

Figure 2.3 shows the current detection circuit. Let the input current $I_{\mathrm{IN}}$ be the current generated by a sensor. Concerning a photodiode, the input current $I_{\mathrm{IN}}$ changes depending on the light intensity. The switch in the upper left of Fig. 2.3 is connected to $V_{\mathrm{CC}}$. As a result of considering the characteristics of an operational amplifier, the relationship between output voltage $V_{\text {OUT }}$ and input current $I_{\text {IN }}$ is shown in Equation (2.2), where $V_{\text {OUT }}$ changes with the change of $I_{\mathrm{IN}}$.

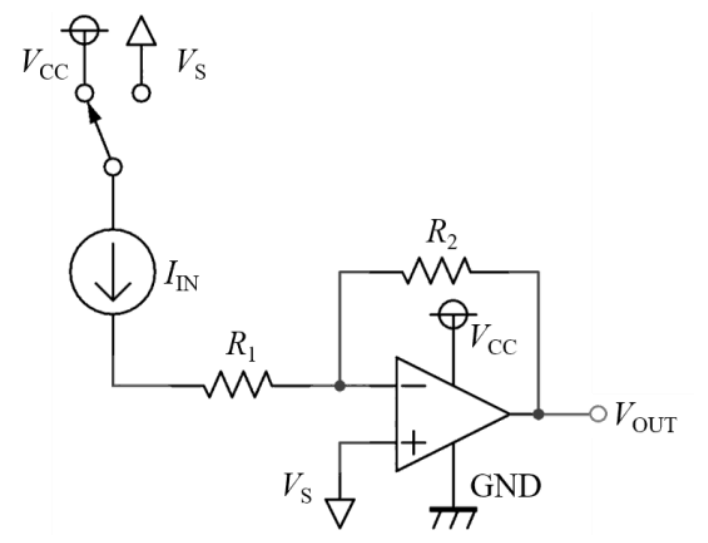

Fig. 2.3 Current detection circuit.

$$
V_{O U T}=-R_{2} I_{I N}+V_{S}
$$

Figure 2.4 shows the resistance detection circuit. Let the resistance $R_{\mathrm{IN}}$ be the resistance value of a sensor. Concerning $\mathrm{CdS}$, the resistance $R_{\mathrm{IN}}$ changes depending on the light intensity. The switch in the upper left of Fig. 2.4 is connected to $V_{\mathrm{CC}}$. As a result of considering the characteristics of an operational amplifier, the relationship between resistance $R_{\mathrm{IN}}$

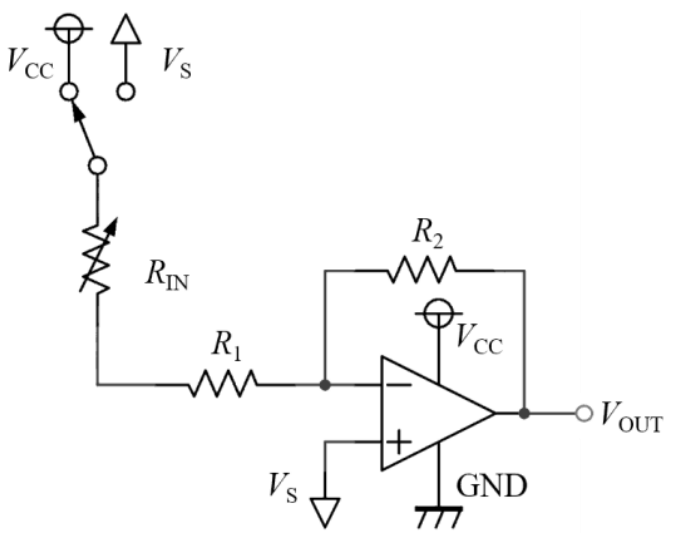

Fig. 2.4 Resistance detection circuit. 
and output voltage $V_{\mathrm{OUT}}$ is defined in Equation (2.3), where it is understood that $V_{\text {OUT }}$ changes with the change of $R_{\mathrm{IN}}$.

$$
V_{\text {OUT }}=-\frac{R_{2}}{R_{I N}+R_{1}}\left(V_{C C}-V_{S}\right)+V_{S}
$$

\section{Experiment and examination}

\subsection{Experimental method}

The output of the proposed analog circuited is imported into the microcomputer board called Arduino with the maximum input of $5 \mathrm{~V}$ through the analog-to-digital conversion. The proposed analog circuit is designed in the range of output voltage from $0 \mathrm{~V}$ to $5 \mathrm{~V}$. The positive power supply of the circuit was $V_{\mathrm{CC}}=5 \mathrm{~V}$, the negative power supply was GND, and $V_{\mathrm{S}}=V_{\mathrm{CC}} / 2=2.5 \mathrm{~V}$.

In this experiment, it is confirmed that three types of units can be detected by replacing three types of sensors connected to the analog circuit. The thermocouple, photodiode, and variable resistor are used as the voltage, current, resistancebased sensors, respectively. The sensors are shown in Fig. 3.1 .

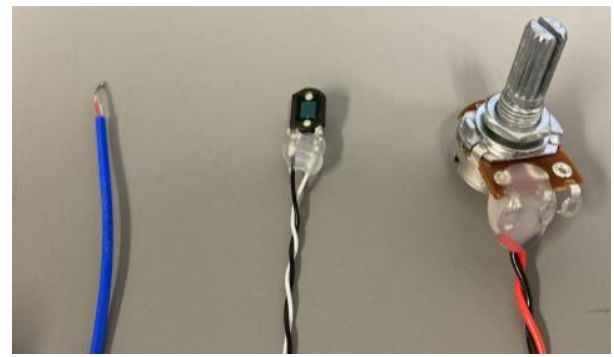

Fig. 3.1 Sensors used in this study.

\subsection{Voltage detection}

Figure 3.2 shows the voltage detection circuit when a thermocouple is used [3]. Let the voltage generated by the thermocouple be $V_{\mathrm{IN}}$. Since $R_{1}=100 \Omega$ and $R_{2}=100 \mathrm{k} \Omega$, the amplification factor is theoretically 1000. A thermocouple was inserted into water, the temperature was changed between 0 and $100^{\circ} \mathrm{C}$, and the output voltage was measured. The result is shown in Fig. 3.3. From this figure, it can be confirmed that the output voltage changes almost linearly with the temperature change. Thus, it was found that the proposed circuit can detect voltage changes. There is an error around $0{ }^{\circ} \mathrm{C}$ because the thermocouple used in this experiment theoretically has an output voltage $V_{\text {OUT }}$ of $2.5 \mathrm{~V}$. The factors causing the error need to be investigated in the future.

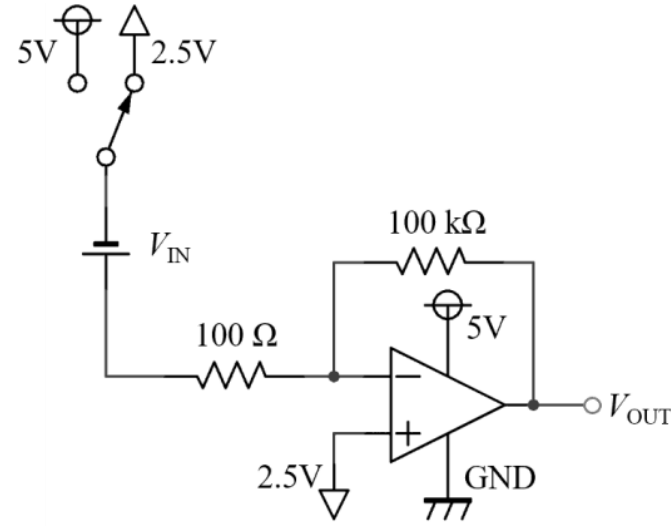

Fig. 3.2 Voltage detection circuit for thermocouples.

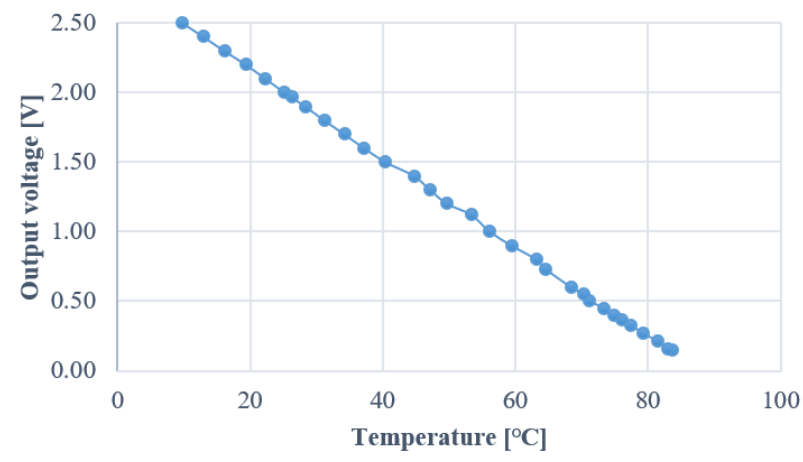

Fig. 3.3 Output voltage vs. temperature.

\subsection{Current detection}

Figure 3.4 shows the current detection circuit using a photodiode. Let the current generated by photodiode be $I_{\mathrm{IN}}$. Here, $R_{1}=100 \Omega$ and $R_{2}=100 \mathrm{k} \Omega$. The illuminance was changed and the output voltage of detection circuit was measured. The illuminance was changed between 0 and 2000 lux. The result is shown in Fig. 3.5. From this figure, the relationship between illuminance and output voltage is considered to be approximately linear. It is supposed that the

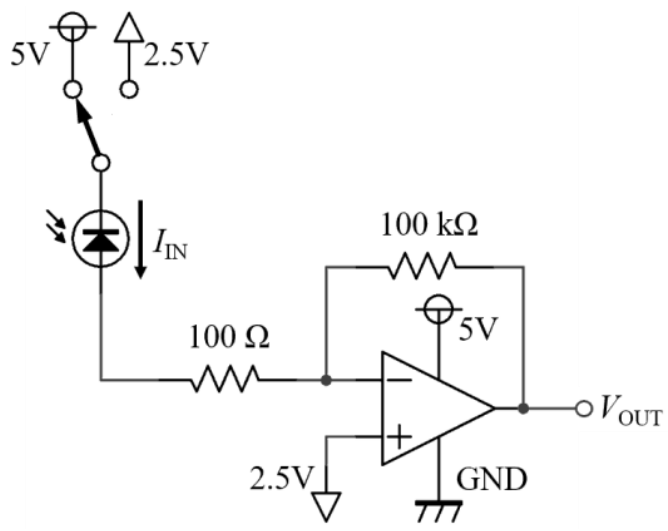

Fig. 3.4 Current detection circuit for photodiode. 
non-linearity is caused by the position of the photodiode and the influence of ambient light. However, since the output voltage changes with the illuminance, it can be seen that the proposed circuit can detect current change.

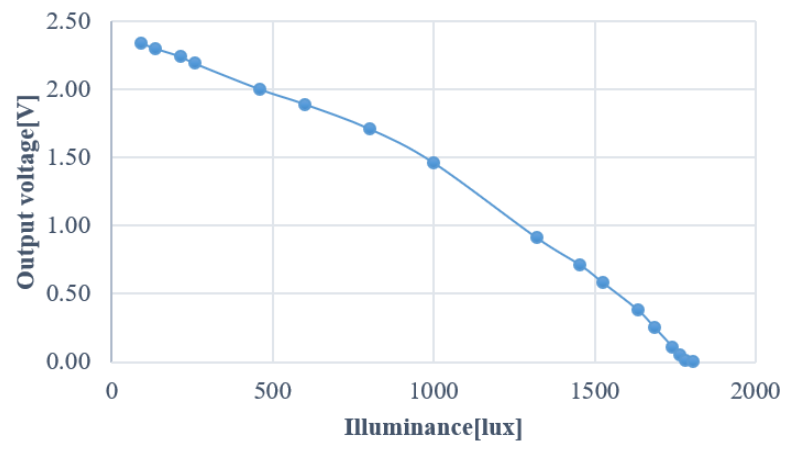

Fig. 3.5 Output voltage vs. illuminance.

\subsection{Resistance detection}

Figure 3.6 shows the resistance detection circuit using a variable resistor. Let the resistance value of the variable resistor be $R_{\mathrm{IN}}$. Here, $R_{1}=100 \Omega$ and $R_{2}=10 \mathrm{k} \Omega$. The output voltage was measured by changing $R_{\mathrm{IN}}$ between 0 and 100 $\mathrm{k} \Omega$. The result is shown in Fig. 3.7. From this figure, it is confirmed that $R_{\mathrm{IN}}$ is in inverse proportion to the output voltage. Since the output voltage changes with the resistance value, it is understood that the resistance change can be detected by the proposed circuit.

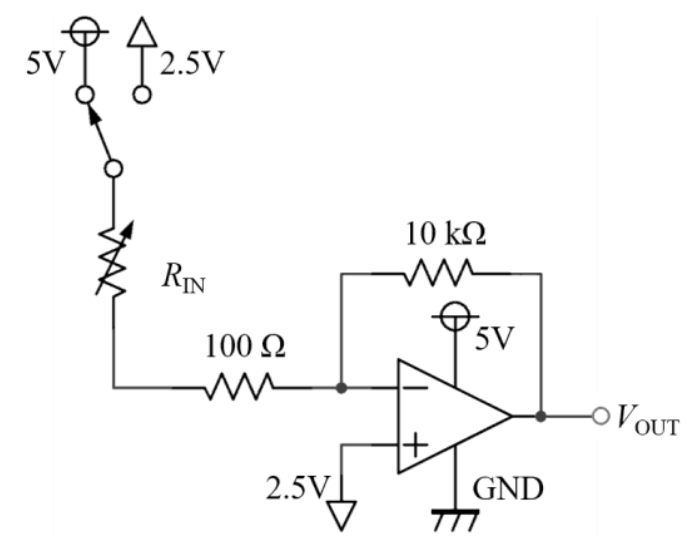

Fig. 3.6 Detection circuit for variable resistance.

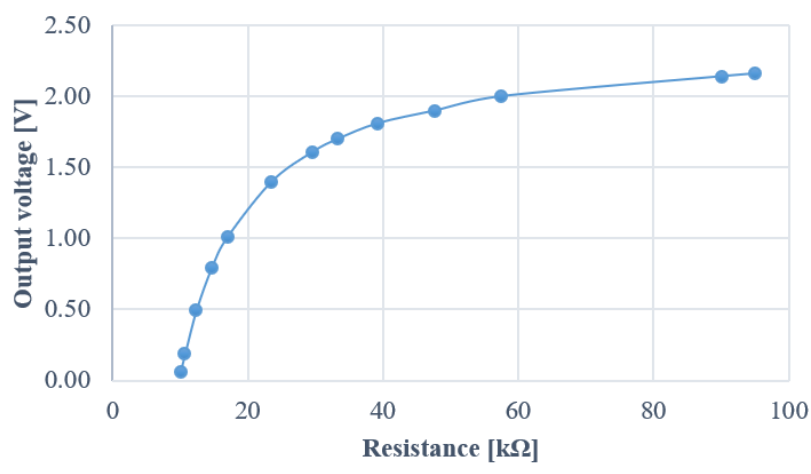

Fig. 3.7 Output voltage vs. resistance.

\section{Conclusions}

The demand for various sensors has been increasing in various fields and requires preparing a detection circuit for each sensor. The sensor-dependent problems include the increase in cost, difficulty in repair and replacement, and different usage depending on the sensor type. To solve those problems, a sensor-independent unique circuit is proposed that can detect electric quantities of voltage, current, and resistance without the prior information on the units of the sensor. It is confirmed that the proposed circuit works with the thermocouple, photodiode, and variable resistor. It is necessary to improve the accuracy of the circuit for practical use. Future works include automatic switching for different sensors.

\section{References}

[1] Ministry of Internal Affairs and Communications, Part 1: Special Feature: IoT, Big Data and AI - New Value Created by Networks and Data, Section 2 Quantitative Verification of Market Size, etc., (3) Sensor, https://www.soumu.go.jp/johotsusintokei/whitepaper/ja /h28/html/nc122530.html (accessed 2021-2-12).

[2] Suematsu, Yasuharu (2015). Introduction to Electronic Circuits, Tokyo : zikkyousyuppan kabusiki gaisya (in Japanese)

[3] Watanabe Electric Corporation. Standard thermal EMF table. https://www.watanabeelectric.co.jp/sensor/faq/netudentui/06.html (accessed 2021-2-12). 